\title{
Towards Multiscale Simulations of Carbon Nanotube Growth Process: A Density Functional Theory Study of Transition Metal Hydrides
}

\author{
Satyender Goel ${ }^{1}$ and Artëm E. Masunov ${ }^{1,2}$ \\ ${ }^{1}$ Nanoscience Technology Center, Department of Chemistry \\ ${ }^{2}$ Department of Physics, \\ University of Central Florida, 12424 Research parkway, Suite 400, Orlando, FL 32826 USA \\ amasunov@mail.ucf.edu
}

\begin{abstract}
Nanoelectronics and photonics applications of single wall carbon nanotubes (SWNT) are feasible only if SWNTs have specific chirality. The knowledge of the detailed mechanism for SWNT synthesis would allow one to optimize the chemical vapor deposition (CVD) process and may help to gain control over selectivity of SWNT synthesis. While it is not probably feasible to study this mechanism experimentally, it could be analyzed using molecular simulations. Here we propose multiscale computer modeling of CVD process. High theory level can be used for di- and tri-atomic fragments, in order to generate parameters for bond order force field. In turn, force field simulations will be used to characterize the chemical origin and thermochemical properties of the intermediates and transition states. This will allow predicting the rate constants for the elementary steps, which are then used in kinetic Monte Carlo simulations to describe SWNT growth at realistic time scales.
\end{abstract}

\section{Introduction}

Single wall carbon nanotubes (SWNT) are cylindrical molecules with unique properties. Many potential applications have been proposed for carbon nanotubes, including conductive and high-strength composites; energy storage and energy conversion devices; sensors; field emission displays and radiation sources; hydrogen storage media; and nanometer-sized semiconductor devices, probes, and interconnects. Some of these applications are now realized in products, others are demonstrated in prototypes. One of the difficulties for nanoelectronic applications of single-walled nanotubes is efficient separation of the nanotubes of different chirality. Increasing chiral selectivity of SWNT synthetic process could solve this problem.

There are successful attempts for selective synthesis of SWNT [1]. Lolli et. al. found that using a CoMo catalyst the chirality distribution of the groeing SWNTs can be reproducibly altered by varying the reaction temperature, the gaseous feed, or the cluster surface morphology. Specifically, increasing the temperature results in increase in nanotube diameter, without a change in the chiral angle. In contrast, by changing the support from $\mathrm{SiO}_{2}$ to $\mathrm{MgO}$, SWNT with similar diameter but different chiral angles are obtained. Clearly, different chirality distributions obtained when varying catalysts support or reaction conditions demonstrate that it is the result of 
chirality-specific differences in the growth kinetics, which in turn depends on the nanotube cap-metal cluster interaction. To fully control SWNT synthesis, one needs guidelines for optimizing the catalyst structure and experimental conditions, which are difficult to obtain without the detailed knowledge of the mechanism for SWNT catalytic synthesis.

Computer simulations may be helpful to establish this mechanism. However, only a few examples are found in the literature that attempt to simulate the SWNT growth. Reactive empirical bond order (REBO) force field was employed to model the catalyzed growth of nanotubes by CVD and investigates nanotube stability as a function of nanotube type, length and diameter through molecular dynamics (MD) approach [2], Since REBO parameters are not available for metals, no catalyst nanoparticles (NP) were considered in this study. The effect of iron cluster size on the structure defects and diameter of the SWNT was reported [3]. Specifically, the study showed that for large particles, containing at least $20 \mathrm{Fe}$ atoms, the caps grow in diameter until they have the same diameter as the cluster. Unfortunately, artificially fast growth rate that made MD simulations feasible, resulted in formation of extremely defective nanotubes. Therefore, it is of interest to simulate catalytic SWNT growth using kinetic models, rather than direct MD approaches. The free energies of the intermediates and reaction barriers need to be obtained, then kinetic Monte Carlo (MC) modeling can be used to determine the effect of reaction conditions on SWNT morphology.

Here we describe multiscale approach to SWNT growth simulation. First, hybrid DFT is used for di- and tri-atomic fragments, to generate parameters for reactive bond order force field. At the next step SWNT/nanocluster systems are constructed, and their geometry is optimized for initial and final structures for different steps of SWNT growth. These structures are then used in MD simulations to identify additional reaction intermediates necessary to build a kinetic model for stepwise SWNT growth. We will start with SWNT having N hexagonal carbon rings in it. The SWNT growth process towards the addition of next ring has number of possibilities through different intermediates with varied reaction rates. Rate constant $k$ for each reactive step will be calculated using Arrhenius equation (1).

$$
k=A e^{-E_{a} / R T}
$$

Here $A$ is the pre-exponential factor, $R$ is the gas constant, $T$ is the temperature, and $E_{a}$ is the activation energy. that is necessery for the reactive system to cross the barrier from one intermediate to the other. A transition states is defined as the state corresponding to the highest energy along this reaction coordinate and is always a first-order saddle point in the energy map. Each transition state can be determined computationally with normal mode analysis by following a specific reaction coordinate corresponding to a single imaginary frequency. When the rate constants of the intermediate formation are known, the kinetic model for catalytic growth can be developed and implemented in Kinetic Monte-Carlo code. Finally, the resultant growth rates calculations using kinetic Monte-Carlo technique will be repeated for SWNT of different chirality. The developed protocol will be used to study SWNT growth with different catalysts under different temperature and feed rate conditions. The specific combinations of these conditions optimized for maximum selectivity will be selected for experimental verification. 
Here, we have investigated bond dissociation energetics for different spin multiplicities in gas-phase neutral hydrides, formed by $3 d$-transition metals from Sc to $\mathrm{Cu}$. Broken-symmetry approach was adapted in order to get the qualitatively correct description of the bond dissociation. The resultant calculations from the current study will be used to parameterize REBO force field for transition metals.

\section{Theoretical Methods}

Density functional theory (DFT) $[4,5]$ in Kohn-Sham formalism combined with approximate exchange-correlation functionals [6] has become a method of choice for the calculation of numerous properties of molecules and solids. Advantage of DFT is considerably lower computational cost as compared to high level multireference $a b$ initio methods of Wave Function Theory (WFT) [7]. Unlike force-field approaches, DFT does not need tedious empirical parameter fitting to produce acceptable results.

In the past decade Transition Metal (TM) hydrides had been used as benchmark system to study efficiency of Density Functional Theory methods. Barone et. al. used pure and hybrid DFT functionals BLYP and B3LYP to study transition metal complexes which includes first row TM hydrides and their cations [8]. B3LYP was found to give accurate dissociation energies, but somewhat overestimate the bond lengths and dipole moments. In a detailed study of $3 d$ transition metal systems including monohydrides Furche and Perdew [9] were not able to reproduce these dissociation energies with the same functionals and basis sets. Presumably, their SCF procedure systematically converged to a different local minimum, as symmetryadapted (SA) unrestricted Kohn-Sham formalism (UKS) was used by Borane et. al. and Broken Symmetry orbitals were used by Furche and Perdew. Among various semilocal (LSDA, BP86, PBE, TPSS) and hybrid density functionals (B3LYP, hTPSS), Furche and Perdew recommend functional TPSS as workhorse of TM compounds. Jensen et. al. [10] has investigated performance of five different density functionals (B3LYP, BP86, PBE0, PBE, BLYP) for diatomics of first row transition metal systems. They concluded that success of a functional is system specific, which means all of these functional are more accurate for certain system and less accurate for others.

Wavefunction theory (WFT) methods were also employed repeatedly to study TM hydrides. WFT uses different approximations to exact solution of the Schrodinger equation given by full configuration interaction (CI) method. Most of them involve multireference SCF procedure (CASSCF or MCSCF) to treat the static correlation, supplemented by double excitations (SDCI or SOCI) to account for dynamic electron correlations. The electronic structure of $\mathrm{NiH}$ was investigated using single reference CI methods three decades ago [11] and, more recently with multireference methods with or without relativistic effects [12-14]. Potential energy curves had been also calculated for other first row TM hydrides, including $\mathrm{TiH}$ [15-17], $\mathrm{CoH}$ [18], $\mathrm{CuH}$ $[14,19], \mathrm{VH}[20]$, and $\mathrm{ScH}$ [21]. Bauschlicher et al. [20] studied the first row TM hydrides (TiH, VH, $\mathrm{CrH}, \mathrm{MnH}, \mathrm{FeH}, \mathrm{NiH})$ using CASSCF/SDCI method. Related method MCSCF+SOCI was used by Koseki et al. to study both ground and excited state PECs of the five first row TM hydrides $(\mathrm{ScH}, \mathrm{TiH}, \mathrm{VH}, \mathrm{CrH}, \mathrm{MnH})$ recently [22-24]. 
Another approximation to full CI was taken by Reiher et. al. [25] to study $\mathrm{CoH}$. They used density matrix renormalization group (DMRG) technique that allows performing CI iteratively without the need to explicitly store any long Slater determinant expansion. DMRG is making study of larger systems involving transition metals feasible, and accurately predict the energy gaps between different spin states.

In this contribution we study diatomic transition metal hydrides, using two DFT functionals and compare their accuracy to the experiment, and WFT results.

\section{Computational Details}

All calculations were done with Gaussian03 program [26] using all-electron Wachters+f basis set [27, 28]. Spin-polarized (unrestricted) DFT was used throughout with no spatial symmetry constraints (broken symmetry, BS). Initial guess was generated by using Harris functional [29] which is the default option in Gaussian03. Self-consistent field (SCF) convergence threshold was set to $10^{-7}$, and relaxed to $10^{-5}$ in a few problematic cases. Initial guess was followed by either geometry optimization or scan along interatomic distance to plot the potential energy curve. In some cases $(\mathrm{CrH}, \mathrm{VH})$ Harris guess lead to SCF convergence problems, and HartreeFock (HF) orbitals were used as a guess. In a few cases where geometry optimization was terminated due to convergence failure, and converged $\mathrm{KS}$ orbitals were used as initial guess (BMK orbitals in case of TPSS non-convergence and vice versa).

A potential complication in the study of systems with nearly degenerate energy levels is the danger of obtaining distinctly different SCF solutions as local energy minima. When different solutions are obtained for the equilibrium geometry and for the dissociation limit, the energy difference is no longer physically meaningful. In order to ensure consistency of SCF solution for all geometries, we built entire potential energy curves and verified that it does not have discontinuities indicating the switch from one SCF solution to another. SCF process was started with fractional occupation numbers (FON) around the Fermi level by using keywords SCF=Fermi and $\operatorname{IOp}(5 / 22=5)$. The occupational numbers became integer at the final SCF cycles. The stability of the SCF solution was checked and KS orbitals were re-optimized (if unstable) using keyword Stable=Opt.

Molden [30] graphical interface was used to examine Kohn-Sham orbitals at the dissociation limit, and at the points where potential curves were found to be nonmonotonic. For one case $(\mathrm{CrH})$ in order to obtain SCF solution with the lower energy, spin-polarization of $\sigma$-bond had to be inverted to have minority spin density localized on $\mathrm{H}$ atom using the keyword Guess=Alter.

\section{Results and Discussion}

\subsection{Dissociation Energies and Dipole Moments}

Potential Energy Curves (PEC) for neutral hydrides for $\mathrm{ScH}, \mathrm{VH}, \mathrm{MnH}$ and $\mathrm{CrH}$ in various multiplicities are reported in Fig.1-4, together with available wave function theory (MCSCF+SOCI) curves. Dissociation energies for neutral hydrides in equilibrium geometry are reported in Table 1. Comparison with experimental data 
[32-38], some of the published Wave Function Theory [22-24, 31] and DFT data [39] are also listed. To calculate the root mean square (rms) deviations for all theoretical values we used experimental data including the error bars. Based on rms values for bond dissociation energies, BMK gives the best agreement with experiment, followed by two WFT methods.

Table 1. Dissociation energies $(\mathrm{kcal} / \mathrm{mol})$ of neutral TM hydrides and root mean square (rms) deviations from the experimental values. Dipole moments (Debye) for neutral metal hydrides calculated with BMK and TPSS, compared with experiment and several WFT levels.

\begin{tabular}{|c|c|c|c|c|c|c|c|c|c|c|c|c|c|c|c|}
\hline Binding Energy & $\mathrm{ScH}$ & & TiH & VH & & $\mathrm{CrH}$ & & & $\mathrm{MnH}$ & & $\mathrm{FeH}$ & $\mathrm{CoH}$ & $\mathrm{NiH}$ & $\mathrm{CuH}$ & \\
\hline Multiplicity & 1 & 3 & 4 & 3 & 5 & 2 & 4 & 6 & 5 & 7 & 4 & 3 & 2 & 1 & rms \\
\hline TPSS & 95.5 & 64.9 & 67.8 & 59.4 & 64.6 & 68.3 & 55.9 & 57.9 & 53.0 & 52.8 & 60.9 & 65.7 & 76.1 & 69.0 & 7.75 \\
\hline BMK & 50.8 & 50.2 & 48.7 & 43.6 & 55.7 & 43.7 & 48.1 & 52.5 & $37.2^{\mathrm{i}}$ & 34.8 & 41.0 & 46.4 & 59.5 & 61.8 & 1.38 \\
\hline $\mathrm{MCSCF}+\mathrm{SOCI}^{\mathrm{a}}$ & 47.3 & & 43.3 & 36.6 & 42.7 & & 37.8 & 44.2 & 21.8 & 33.6 & & & & & 1.84 \\
\hline $\mathrm{MCPF}^{\mathrm{b}}$ & 51.0 & & 47.3 & & 53.0 & & & 48.7 & 21.9 & 39.4 & 45.0 & 44.7 & 64.3 & 61.6 & 1.63 \\
\hline 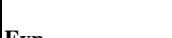 & 47.5 & & 48.9 & & 49.1 & & & 44.5 & & 30.2 & 37.5 & 46.0 & 59.4 & 61.0 & \\
\hline Exp. & $\pm 2.0^{\mathrm{c}}$ & & $\pm 2.1^{\mathrm{d}}$ & & $\pm 1.6^{\mathrm{e}}$ & & & $\pm 1.6^{\mathrm{e}}$ & & $\pm 4.4^{\mathrm{f}}$ & $\pm 1.9^{\mathrm{g}}$ & $\pm 3.0^{\mathrm{h}}$ & $\pm 3.0^{\mathrm{h}}$ & $\pm 4.0^{\mathrm{h}}$ & \\
\hline \multicolumn{16}{|l|}{ Dipole Moments } \\
\hline BMK & 1.870 & 2.600 & 2.570 & 2.000 & 2.654 & 2.500 & 1.800 & 3.100 & 1.379 & 1.106 & 2.576 & 2.471 & 3.165 & 2.952 & \\
\hline TPSS & 2.530 & 2.800 & 2.616 & 2.100 & 2.497 & 2.900 & 2.600 & 2.905 & 2.053 & 0.756 & 2.630 & 2.566 & 2.304 & 2.468 & \\
\hline $\mathrm{SDCI}^{\mathrm{j}}$ & 1.421 & 1.959 & 1.899 & & 1.788 & & & 4.250 & & 1.296 & 4.098 & 3.895 & 3.676 & 3.880 & \\
\hline $\mathrm{CPF}^{\mathrm{j}}$ & 1.776 & 2.554 & 2.308 & & 2.319 & & & 3.779 & & 1.227 & 1.311 & 1.448 & 1.806 & 2.749 & \\
\hline $\mathrm{MCPF}^{\mathrm{j}}$ & 1.641 & 2.432 & 2.185 & & 2.021 & & & 3.807 & & 1.239 & 2.901 & 2.743 & 2.557 & 2.951 & \\
\hline Exp. & & & $2.455^{\mathrm{k}}$ & & & & & $\begin{array}{c}3.510^{1}( \\
\mathrm{CrD})\end{array}$ & & & $2.630^{\mathrm{m}}$ & & $2.440^{\mathrm{n}}$ & & \\
\hline
\end{tabular}

${ }^{\mathrm{a}}$ Ref.[22-24], ${ }^{\mathrm{b}}$ Ref.[31], ${ }^{\mathrm{c}}$ Ref.[32], ${ }^{\mathrm{d}}$ Ref.[33], ${ }^{\mathrm{c}}$ Ref.[34], ${ }^{\mathrm{f}}$ Ref.[35], ${ }^{\mathrm{g}}$ Ref.[36], ${ }^{\mathrm{h}}$ Ref.[37-38], ${ }^{\mathrm{i}}$ Ref.[39],

${ }^{\mathrm{j}}$ Ref.[40], ${ }^{\mathrm{k}}$ Ref.[41], ${ }^{1}$ Ref.[42], ${ }^{\mathrm{m}}$ Ref.[43], ${ }^{\mathrm{n}}$ Ref.[44]

Dipole moments calculated with BMK and TPSS functionals are also reported in Table 1 and compared to the experimental data reported by Steimle et al. [41-44] and WFT results compiled by Chong et al. [40]. One can see from the Table 1, BMK and TPSS values for the dipole moments are in close agreement with experiment for $\mathrm{TiH}$, $\mathrm{FeH}$ and $\mathrm{CrD}$. For $\mathrm{NiH}$ the TPSS is in much better agreement with experiment, than BMK ones. For $\mathrm{NiH}$ and $\mathrm{CoH}$ the WFT results are in strong disagreement with each other, which indicated the severe difficulties in description of the electronic structure of these molecules. This clearly merits the future investigation.

\subsection{Potential Energy Curves and Spin Gaps}

The potential energy curves for TM hydrides are plotted on Fig. 1-4. The first two lowest multiplicities for $\mathrm{ScH}$ (Fig 1) are close in energy but differ in the bond length, so that singlet is more stable at the shorter, and triplet at longer bond length according to BMK results. On the contrary, TPSS overstabilizes singlet at all distances. Only singlet multiplicity is reported in the previous works [31, 9, 21, 45] including WFT study by Koseki et. al. [23] 


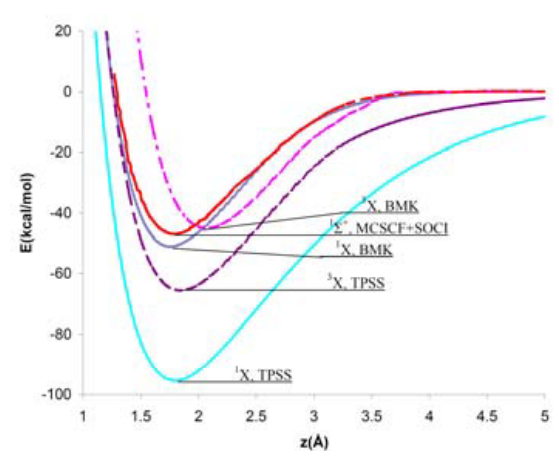

Fig. 1. Potential Energy Curves of $\mathrm{ScH}$ with multiplicity 1 and 3, calculated by TPSS, BMK, and WFT [23] methods

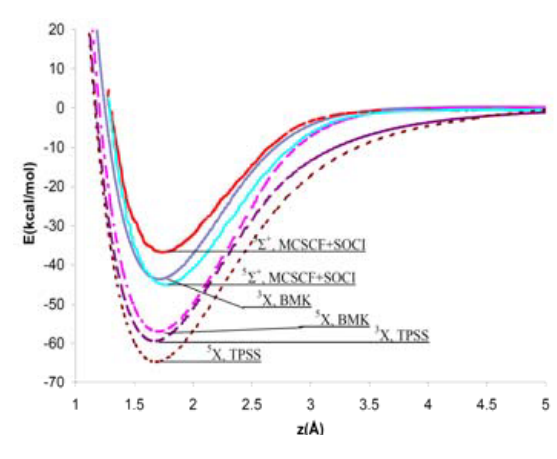

Fig. 2. Potential Energy Curves of VH with multiplicity 3 and 5 , calculated by TPSS, BMK, and WFT [24] methods

Two spin multiplicities for VH are reported on Fig 2 at WFT level [24]. Both BMK and TPSS reproduce this ordering, although the spin gap in BMK is twice larger than in WFT. BMK result seems to be more reliable as it closely reproduces experimental $\mathrm{D}_{\mathrm{e}}$ for the multiplicity 5 [34]. The two multiplicities for $\mathrm{MnH}$ (Fig 3) are almost degenerate in both BMK and TPSS, while WFT favors $\mathrm{M}=7$ by $11 \mathrm{kcal} / \mathrm{mol}$. Three Multiplicities of $\mathrm{CrH}$ are reported at WFT [3] (Fig 4), all with different dissociation limits. Both WFT and DFT predict the ground state to have the multiplicity of 6 . BMK and especially TPSS underestimate the spin gap at equilibrium, as compared to the WFT predictions, while reproduce it fairly well at the dissociation limit.

The energy difference between the high and low spin states was studied previously by several authors and found to depend strongly on the fraction of HF exchange. This can be attributed to the fine balance between the negative HF exchange energy contribution from the electron of the same spin, which is opposite in sign to the electronic correlation contribution arising from the repulsion between any two electrons regardless of their spin. A method which includes the exchange and neglects the correlation (such as HF) will favor high multiplicities by maximizing the number of electrons with the same spin. On the contrary, self-interaction error in pure DFT favors low-spin states. Attempts to improve the relative spin-state energies description of density functionals include hybrid DFT schemes as well as DFT+U [46]. It was recently shown that $\mathrm{DFT}+\mathrm{U}$ is capable of providing the qualitatively correct splitting in low- and high-spin iron porphyrins [46] and $\mathrm{FeO}^{+}$[47]. However, when hybrid DFT approach is adapted, the accurate spin energy gaps can be obtained by adjusting fraction of HF exchange in DFT functional [48]. Conradie and Ghosh [49] studied $\mathrm{Fe}(+2)$ spin-crossover complexes and found that pure functionals such as BLYP, PW91 and BP86 incorrectly favor spin-coupled form (covalent description), while hybrid functionals such as B3LYP lean in the other direction. To correct the latter, they suggested using B3LYP* [50-52] with reduced amount of Hartree-Fock exchange in B3LYP from the standard 20\% to 15\%; the B3LYP* functional has been found to 


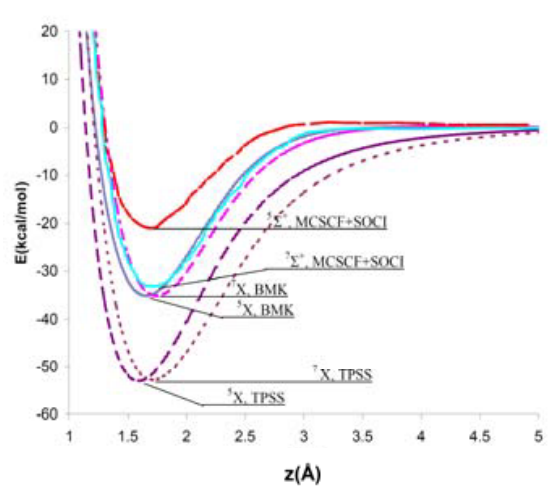

Fig. 3. Potential Energy Curves of $\mathrm{MnH}$ with multiplicity 5 and 7, calculated by TPSS, BMK, and WFT [22] methods

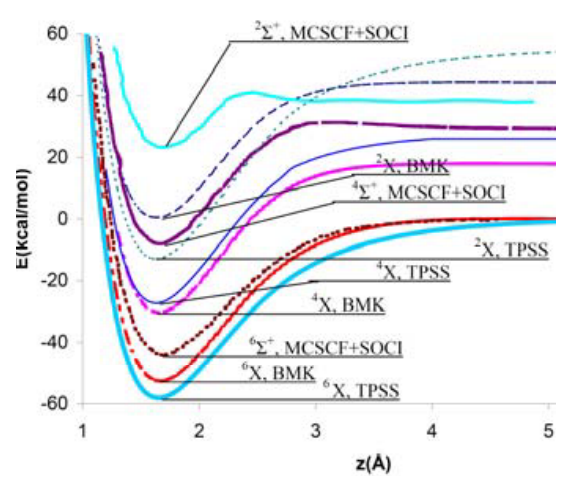

Fig. 4. Potential Energy Curves of $\mathrm{CrH}$ with multiplicity 2,4 and 6 , calculated by non relativistic TPSS, BMK, and WFT (14) methods

give improved results. Harvey [53] also found an 15\% fraction of exact exchange yields accurate results in many other cases. It appears that the large fraction of HF exchange is necessary for correct prediction of the dissociation energies, while smaller fraction is in better agreement with experimentally observed spin-gaps.

\section{Conclusion}

As a first step in design of the reactive bond order forcefield for TM compounds, we used two exchange-correlation functionals including explicit dependence on the kinetic energy density ( $\boldsymbol{\tau}$-functionals) to study neutral hydrides formed by $3 d$ transition metals $(\mathrm{Sc}-\mathrm{Cu})$. One of the functionals selected contained large fraction of Hartree-Fock exchange (BMK), and another one was a pure DFT functional (TPSS). Watchers basis sets [28], augmented with $f$-functions by Hay et. al. [29] were used. We have taken particular care to obtain the consistent SCF solution, including the stability analysis and Fermi smearing. In order to ensure the stability of Slater determinant in the entire range of interatomic distances, the potential energy curves were plotted and inspected for discontinuities. When found, the discontinuities were eliminated by using the lower energy orbitals as initial guess to continue the curve smoothly. The spin orbitals at the dissociation limit were inspected and reordered if necessary.

Qualitatively correct description of the bond dissociation was ensured by allowing the spatial and spins symmetry to break, which resulted in some spin-contamination for several systems at equilibrium. However, our calculated BMK dissociation energies are in better agreement with experiment than those obtained with high level wavefunction theory methods from the literature. The next step in this study will be the validation of BMK functional for the metal-metal and metal-carbon potential curves. These curves will be used to calibrate parameters for the reactive bond order force field and the subsequent Molecular Dynamics study of SWNT/metal growth process. 


\section{Acknowledgements}

This work is supported in part by UCF start up grant. SG gratefully acknowledges Interdisciplinary Information Science Laboratory (I2lab) fellowship. The computer time was generously provided by Stokes HPC facilities at UCF Institute for Simulation and Training (IST), UCF I2lab, and DOE NERSC.

\section{References}

1. Lolli, G., et al.: Tailoring $(\mathrm{n}, \mathrm{m})$ structure of single-walled carbon nanotubes by modifying reaction conditions and the nature of the support of CoMo catalysts. J. Phys. Chem. B. 110, 2108-2115 (2006)

2. Sinnott, S.B., et al.: Model of carbon nanotube growth through chemical vapor deposition. Chem. Phys. Lett. 315, 25-30 (1999)

3. Ding, F., Rosen, A., Bolton, K.: Molecular dynamics study of the catalyst particle size dependence on carbon nanotube growth. J. Chem. Phys. 121, 2775-2779 (2004)

4. Kohn, W., et al.: Density functional theory of electronic structure. J. Phys. Chem. 100, 12974-12980 (1996)

5. Kohn, W., Sham, L.J.: Self-Consistent Equations Including Exchange and Correlation Effects. Phys. Rev. 140, 1133 (1965)

6. Geerlings, P., et al.: Conceptual density functional theory. Chem. Rev. 103, 1793-1873 (2003)

7. Pettersson, L.G.M., et al.: Positive-Ions of the 1st-Row and 2nd-Row Transition-Metal Hydrides. J. Chem. Phys. 87, 481-492 (1987)

8. Barone, V., et al.: Comparison of conventional and hybrid density functional approaches. Cationic hydrides of first-row transition metals as a case study. Chem. Phys. Lett. 249, 290-296 (1996)

9. Furche, F., Perdew, J.P.: The performance of semilocal and hybrid density functionals in 3d transition-metal chemistry. J. Chem. Phys. 124, 27 (2006)

10. Jensen, K.P.: Performance of density functionals for first row transition metal systems. J. Chem. Phys. 126, 14 (2007)

11. Bagus, P.S., et al.: Electronic-Structure of Transition-Metal Hydrides - NiH and PdH. Phys. Rev. A. 23, 461-472 (1981)

12. Blomberg, M.R.A., et al.: A Theoretical-Study of NiH - Optical-Spectrum and Potential Curves. Mol. Phys. 47, 127-143 (1982)

13. Marian, C.M., et al.: Multireference and Relativistic Effects in NiH. J. Chem. Phys. 91, 3589-3595 (1989)

14. Pouamerigo, R., et al.: The Chemical-Bonds in $\mathrm{CuH}, \mathrm{Cu}-2, \mathrm{NiH}$, and Ni-2 Studied with Multiconfigurational 2nd-Order Perturbation-Theory. J. Chem. Phys. 101, 4893-4902 (1994)

15. Anglada, J., et al.: Theoretical Investigation of the Low-Lying Electronic States of TiH. Mol. Phys. 69, 281-303 (1990)

16. Bauschlicher, C.W.: Full Configuration-Interaction Benchmark Calculations for TiH. J. Phys. Chem. 92, 3020-3023 (1988)

17. Dai, D.G., et al.: Spectroscopic Properties and Potential-Energy Curves for 21 Electronic States of CrH. J. Mol. Spec. 161, 455-465 (1993)

18. Freindorf, M., Marian, C.M., Hess, B.A.: Theoretical-Study of the Electronic-Spectrum of the CoH Molecule. J. Chem. Phys. 99, 1215-1223 (1993) 
19. Raghavachari, K., Sunil, K.K., Jordan, K.D.: Theoretical-Study of the Bonding in $\mathrm{CuH}$ and $\mathrm{Cu}_{2}$. J. Chem. Phys. 83, 4633-4640 (1985)

20. Walch, S.P., Bauschlicher, C.W.: CASSCF/Cl Calculations for 1st Row Transition-Metal Hydrides - the TiH (4-Phi), VH (5-Delta), CrH (6-Sigma+), MnH (7-Sigma+), FeH (4,6Delta), and NiH (2-Delta) States. J. Chem. Phys. 78, 4597-4605 (1983)

21. Kunz, A.B., Guse, M.P., Blint, R.J.: Potential-Energy Curves for ScH. J. Phys. B-Atom. Mol. Opt. Phys. 8, L358-L361 (1975)

22. Koseki, S., Matsushita, T., Gordon, M.S.: Dissociation potential curves of low-lying states in transition metal hydrides. 3. Hydrides of groups 6 and 7. J. Phys. Chem. A. 110, 25602570 (2006)

23. Koseki, S., et al.: Dissociation potential curves of low-lying states in transition metal hydrides. I. Hydrides of Group 4. J. Phys. Chem. A. 106, 785-794 (2002)

24. Koseki, S., et al.: Dissociation potential curves of low-lying states in transition metal hydrides. 2. Hydrides of groups 3 and 5. J. Phys. Chem. A. 108, 4707-4719 (2004)

25. Marti, K.H., et al.: Density matrix renormalization group calculations on relative energies of transition metal complexes and clusters. J. Chem. Phys. 128 (2008)

26. Frisch, M.J., et al.: GAUSSIAN03, Rev. D1. Gaussian Inc., Wallingford (2004)

27. Wachters, A.J.: Gaussian Basis Set for Molecular Wavefunctions Containing Third-Row Atoms. J. Chem. Phys. 52, 1033 (1970)

28. Hay, P.J.: Gaussian Basis Sets for Molecular Calculations - Representation of 3d Orbitals in Transition-Metal Atoms. J. Chem. Phys. 66, 4377-4384 (1977)

29. Harris, J.: Simplified Method for Calculating the Energy of Weakly Interacting Fragments. Phys. Rev. B. 31, 1770-1779 (1985)

30. Schaftenaar, G., Noordik, J.H.: Molden: a pre- and post-processing program for molecular and electronic structures. J. Comp.-Aid. Mol. Des. 14, 123-134 (2000)

31. Harrison, J.F.: Electronic structure of diatomic molecules composed of a first-row transition metal and main-group element (H-F). Chem. Rev. 100, 679-716 (2000)

32. Kant, A., Moon, K.A.: Mass-Spectrometric Determination of the Dissociation-Energies of Gaseous $\mathrm{AiH}, \mathrm{GaH}, \mathrm{InH}, \mathrm{ScH}$, and $\mathrm{CoH}$ and Estimation of the Maximum DissociationEnergies of TlH, CrH, MnH, and FeH. High Temp. Science 14, $23-31$ (1981)

33. Chen, Y.M., Clemmer, D.E., Armentrout, P.B.: The Gas-Phase Thermochemistry of TiH. J. Chem. Phys. 95, 1228-1233 (1991)

34. Chen, Y.M., Clemmer, D.E., Armentrout, P.B.: Gas-Phase Thermochemistry of VH and CrH. J. Chem. Phys. 98, 4929-4936 (1993)

35. Sunderlin, L.S., Armentrout, P.B.: Reactions of Mn+ with I-C4H10, Neo-C5H12 $\left(\mathrm{CH}_{3}\right)_{2} \mathrm{CO}$, Cyclo- $\mathrm{C}_{3} \mathrm{H}_{6}$, and Cyclo- $\mathrm{C}_{2} \mathrm{H}_{4} \mathrm{O}-$ Bond-Energies for $\mathrm{MnCH}_{2}+, \mathrm{MnH}$, and $\mathrm{MnCH}_{3}$. J. Phys. Chem. 94, 3589-3597 (1990)

36. Schultz, R.H., Armentrout, P.B.: The Gas-Phase Thermochemistry of FeH. J. Chem. Phys. 94, 2262-2268 (1991)

37. Kant, A., Moon, K.A.: Dissociation-Energies of Gaseous $\mathrm{CuH}, \mathrm{AgH}, \mathrm{AuH}$, and NiH. High Temp. Science. 11, 52-62 (1979)

38. Fisher, E.R., Armentrout, P.B.: Reactions of $\mathrm{Co}+\mathrm{Ni}+$, and $\mathrm{Cu}+$ with $\mathrm{Cyclopropane}$ and Ethylene-Oxide - Metal Methylidene Ion Bond-Energies. J. Phys. Chem. 94, 1674-1683 (1990)

39. Goel, S., Masunov, A.E.: Potential energy curves and electronic structure of $3 \mathrm{~d}$ transition metal hydrides and their cations. J. Chem. Phys. 129, 214302-214314 (2008)

40. Chong, D.P., Langhoff, S.R., Bauschlicher, C.W., Walch, S.P.: Theoretical DipoleMoments for the 1st-Row Transition-Metal Hydrides. J. Chem. Phys. 85, 2850-2860 (1986) 
41. Chen, J., Steimle, T.C.: A molecular beam optical Stark study of nickel monohydride, $\mathrm{NiH}$. Chem. Phys. Lett. 457, 23-25 (2008)

42. Steimle, T.C., Shirley, J.E., Simard, B., Vasseur, M., Hackett, P.: A laser spectroscopic study of gas-phase TiH. J. Chem. Phys. 95, 7179 (1991)

43. Chen, J., Steimle, T.C., Merer, A.J.: The permanent electric dipole moment of chromium monodeuteride, CrD. J. Chem. Phys. 127, 204307 (2007)

44. Steimle, T.C., Chen, J., Harrison, J., Brown, J.M.: A molecular-beam optical Stark study of lines in the $(1,0)$ band of the $\mathrm{F}^{4} \Delta_{7 / 2}-\mathrm{X}^{4} \Delta_{7 / 2}$ transition of iron monohydride, FeH. J. Chem. Phys. 124, 184307 (2006)

45. Barone, V., Adamo, C.: First-row transition-metal hydrides: A challenging playground for new theoretical approaches. Int. J. Quan. Chem. 61, 443-451 (1997)

46. Scherlis, D.A., et al.: Simulation of heme using DFT+U: A step toward accurate spin-state energetics. J. Phys. Chem. B. 111, 7384-7391 (2007)

47. Kulik, H.J., et al.: Density functional theory in transition-metal chemistry: A selfconsistent Hubbard U approach. Phys. Rev. Lett. 97 (2006)

48. Sorkin, A., Iron, M.A., Truhlar, D.G.: Density functional theory in transition-metal chemistry: Relative energies of low-lying states of iron compounds and the effect of spatial symmetry breaking. J. Chem. Theo. Comp. 4, 307-315 (2008)

49. Conradie, J., Ghosh, A.: DFT calculations on the spin-crossover complex Fe(salen)(NO): a quest for the best functional. J. Phys. Chem. B. 111, 12621-12624 (2007)

50. Reiher, M.: Theoretical study of the Fe(phen)(2)(NCS)(2) spin-crossover complex with reparametrized density functionals. Inor. Chem. 41, 6928-6935 (2002)

51. Salomon, O., Reiher, M., Hess, B.A.: Assertion and validation of the performance of the B3LYP* functional for the first transition metal row and the G2 test set. J. Chem. Phys. 117, 4729-4737 (2002)

52. Reiher, M., Salomon, O., Hess, B.A.: Reparameterization of hybrid functionals based on energy differences of states of different multiplicity. Theo. Chem. Acc. 107, 48-55 (2001)

53. Harvey, J.N.: DFT computation of relative spin-state energetics of transition metal compounds. In: Principles and Applications of Density Functional Theory, Inorg. Chem., pp. 151-183 (2004) 